\title{
Effect of Repeated Sessions of Transcranial Direct Current Stimulation on Functional Balance in Parkinson's Disease: A Pilot Randomized Controlled Study
}

\author{
Bijan Forogh ${ }^{1}$, Seyed Pezhman Madani ${ }^{1}$ Maryam Rafiei ${ }^{2}$, Mohammad R Motamed ${ }^{3}$ and Simin \\ Sajadi $4^{*}$
}

${ }^{1}$ Department of Physical Medicine and Rehabilitation, Neuromusculoskeletal Research Center, Fatemeh Zahra Hospital, Iran University of Medical Sciences, Tehran, Iran

${ }^{2}$ Department of Physical Medicine and Rehabilitation, Imam Khomeini Hospital, Khomein, Iran

${ }^{3}$ Department of Neurology, Firoozgar Hospital, Iran University of Medical Sciences, Tehran, Iran

${ }^{4}$ Department of Physical Medicine and Rehabilitation, Neuromusculoskeletal Research Center, Shafa Yahyaian Hospital, Iran University of Medical Sciences, Tehran, Iran

*Corresponding author: Simin Sajadi, MD, Neuromusculoskeletal Research Centre, Shafa Yahyaian Hospital, Baharestan Square, Tehran, 1157637131, Islamic Republic of Iran, Tel: (+98) 21 33542001; Fax: (+98) 21 33542020; E-mail: dr.sajadi_pmr@yahoo.com

Rec Date: November 04, 2017; Acc Date: March 05, 2018, 2018; Pub Date: March 07, 2018

Citation: Forogh B, Madani SP, Rafiei M, Motamed MR, Sajadi S (2018) Effect of Repeated Sessions of Transcranial Direct Current Stimulation on Functional Balance in Parkinson's Disease: A Pilot Randomized Controlled Study. J Neurol Neurosci Vol.9 No.2:250.

\section{Abstract}

Background: Trans cranial direct current stimulation is a new treatment for neuromodulation and in several studies showed positive effect on Parkinson's disease especially motor symptoms. The aim of this study was to assess additive effects of consecutive sessions of tDCS on functional balance in patients with PD.

Method: In this pilot randomized double blind parallel study, 23 patients with Parkinson's disease (PD) divided in two groups of real Trans Cranial Direct Current Stimulation plus occupational therapy and sham Trans Cranial Direct Current Stimulation plus occupational therapy and the effects of therapeutic sessions ( 8 sessions Trans Cranial Direct Current Stimulation with $0.6 \mathrm{~mA} / \mathrm{cm}^{2}$ current, 20 minute on dorsolateral prefrontal cortex ) were evaluated on balance just after therapeutic course and in 3 months follow-up.

Results: There was no significant time-Group interaction in any time point showing that behavior of experimental and sham groups didn't differ regarding changes in BBS ( $d f=1.44, F=0.91, p=0.38$ ).

Conclusion: Based on the results of the present study there is no significant effect of tDCS on functional balance in patients with PD at any time point.

Keywords: Transcranial direct current stimulation; Parkinson's disease; Balance; Occupational therapy

\section{Introduction}

Parkinson's disease (PD) is a progressive neurodegenerative disorder, primarily characterized by motor symptoms (MS) such as tremor, rigidity, bradykinesia, stiffness, slowness and impaired equilibrium [1] and non- motor symptoms (NMS) include fatigue, autonomic dysfunction, cognitive/ neurobehavioral disorders, and sensory and sleep abnormalities [2]. Postural instability is one of the cardinal symptoms of PD and Studies have shown subclinical manifestation of postural instability and balance dysfunction in the early stage of PD [3].

There is a high fall incidence, even in optimally-medicated, early-stage PD (40-70\%). However, balance problems and resulting falls are major factors determining quality of life, morbidity, and mortality in individuals with PD [4,5]. Impairment of postural control in Patients with PD is due to different causes including rigidity, bradykinesia of postural responses, impaired kinesthesia for sensory integration, bradykinetic gait with freezing, and less automaticity of gait and balance [5]. Balance dysfunction is a clinical concern since they are not easily amenable to treatment neither with medication nor by surgical method in the form Deep brain stimulation $[6,7]$.

Transcranial direct current stimulation (TDCS) is a noninvasive technique for inducing prolonged functional changes in the human cerebral cortex [8]. Previous studies have shown the benefit of TDCS on motor function, bradykinesia, gait, working memory, executive function and fatigue in patients with PD [9-14].

Significant motor improvement after right dorsolateral prefrontal cortex (DLPFC) stimulation [10] and reduced bradykinesia, and increased walking speed after TDCS of the motor and prefrontal cortices have been reported [9]. Also, 
anodal stimulation of TDCS has produced significant improvement of gait with reduction in number and duration of freezing of gait episodes along with a significant reduction in the Unified Parkinson's Disease Rating Scale score [11].

TDCS can modulate cortical excitability and prefrontal dopamine release and enhance neurophysiological mechanisms that compensate for impaired learning in PD and affect motor performance. TDCS has the potential to enhance rehabilitation effects in the elderly and in patients with neurological diseases $[13,15,16]$. However, preliminary evidence regarding benefit of combining TDCS and physical training on gait $[17,18]$ and effect of TDCS on balance is conflicting $[18,19]$. The aim of this study was to assess additive effects of consecutive sessions of TDCS on functional balance in patients with PD.

\section{Materials and Methods}

\section{Study setting}

This pilot double-blind controlled trial was conducted at Physical Medicine and Rehabilitation department, Firoozgar Hospital in Tehran, Iran.

\section{Participants}

Eligible participants were Twenty three patients ( 9 women and 14 men) aged between 36 and 80 years (mean age 63 years) with idiopathic Parkinson's who were in stage 2 or 3 of Parkinson disease based on Hoehn and Yahr criteria diagnosed by a neurologist; were under stable pharmacological regime at least 30 days before entrance the study; have stable clinical condition; have a good primary response to Levo-DOPA or DOPA agonists; have normal MRI.

Patients excluded from the study if they have have dementia related to Parkinson's disease using Mini-Cog test and also, have parkinsonism related to drugs, history of epilepsy, any other neurological disorder or have metal implants in the head including deep brain stimulator or aneurysm clips. The study protocol was approved by the local ethics committee at Iran University of Medical Sciences and underwent in accordance with the ethical standards of the Helsinki Declaration. The procedure was explained to the patients and they signed written informed consent before participation in the study.

\section{Intervention}

Patients were allocated into real or sham treatment groups using a simple randomization method. Each patient received eight sessions of real or sham anodal tDCS in two weeks and occupational therapy just after each session.

12 patients were assigned to real tDCS group and 11 patients to sham tDCS group. Irrespective of more involved side of body, the anode electrode was positioned over left DLPFC area that is localized as $5 \mathrm{~cm}$ in front of $\mathrm{C} 1$ using international 10-20 electroencephalogram system. Cathode electrode was placed over Right DLPFC.

A battery driven stimulator (Activadose II) generated direct electrical current with a maximum current output of 4 milliampere. Two pairs of $35 \mathrm{~cm}^{2}$ rubber electrodes covered with $0.9 \%$ saline soaked sponges were used for transmission.

In both groups the direct current was ramped up to 0.6 $\mathrm{mA} / \mathrm{cm}^{2}$ during $30 \mathrm{~s}$. Experimental group received $20 \mathrm{~min}$ of real stimulation with a current intensity of $0.6 \mathrm{~mA} / \mathrm{cm}^{2}$. After the initial ramp-up in sham group, the current was directly ramped down to 0 and patients and after initial tingling sensation patients didn't receive any stimulation in the remaining time. The ramp-down time was 4 seconds in both groups. The stimulator was placed out of sight of patients considering blindness.

\section{Outcome}

Berg Balance Scale (14 items, each 0-4, total score is mean of 14 items range $0-4$, lower score_ increasing severity) was used as outcome measure to evaluate patient functional balance at baseline, just after treatments (after two weeks) and 3 months later. Participants and rater were both blinded to the treatment

\section{Statistical analysis}

Data analysis was performed by SPSS 22 software. A Greenhouse-Geisser correction was used for sphericity violation. "Kolmogorov Smirnov" test showed data had normal distribution so, parametric tests were used. Independent sample t-test and Chi-square test were used for analysis of Baseline characteristic. "Mixed design ANOVA" was used to explore the main and interaction effects of time and group on BBS. Statistical significance was defined at $<0.05$.

\section{Results}

A total of 40 patients were evaluated and based on inclusion criteria, 23 patients were enrolled in the study and all of them completed study. These patients (14 males and 9 females) were randomly allocated to real/sham groups. Baseline characteristic of experiment and sham groups are shown in Table 1.

Table 1 Baseline characteristics of experimental and sham groups.

\begin{tabular}{|l|l|l|l|}
\hline Variables & Experimental (N=12) & Sham (N=11) & $\begin{array}{l}\text { P- } \\
\text { value }\end{array}$ \\
\hline Age (years) & 61.33 & 64.81 & $0.478^{\mathrm{a}}$ \\
\hline Gender (N) & 7 female & 7 female & $1.000^{\mathrm{b}}$ \\
\hline BBS (mean $\pm \mathrm{SD})$ & $45.08 \pm 15.95$ & $50.54 \pm 9.61$ & $0.337^{\mathrm{a}}$ \\
\hline
\end{tabular}

Although Berg Balance Scale didn't change significantly in either group at any time point but the main effect of time was significant in a whole sample (experiment and sham groups) 
between before and just after therapeutic course termination $(p=0.02)$ but not after 3 months follow up (Table 2$)$.

Table 2 Pair wise comparison of BBS in whole sample.

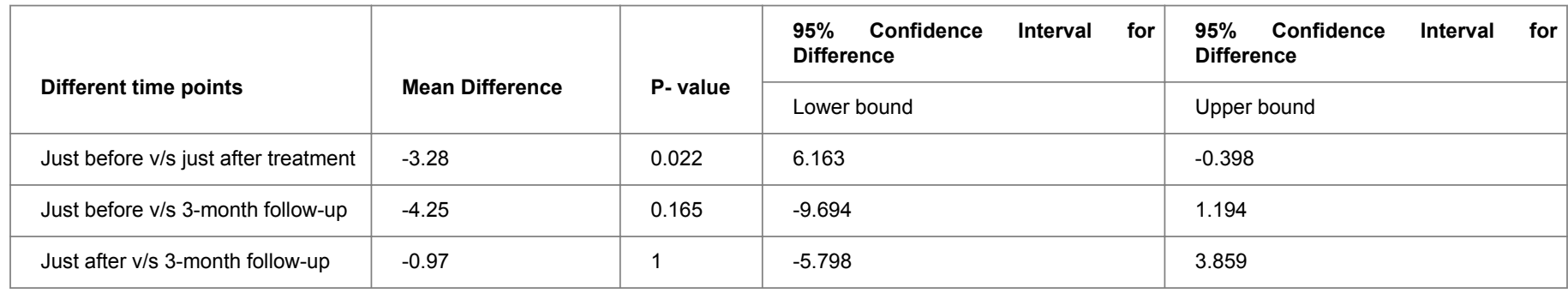

There was no significant time-Group interaction in any time point and behavior of groups didn't differ regarding changes in BBS $(\mathrm{df}=1.44, \mathrm{~F}=0.91, \mathrm{p}=0.38$ ) (Figure 1).

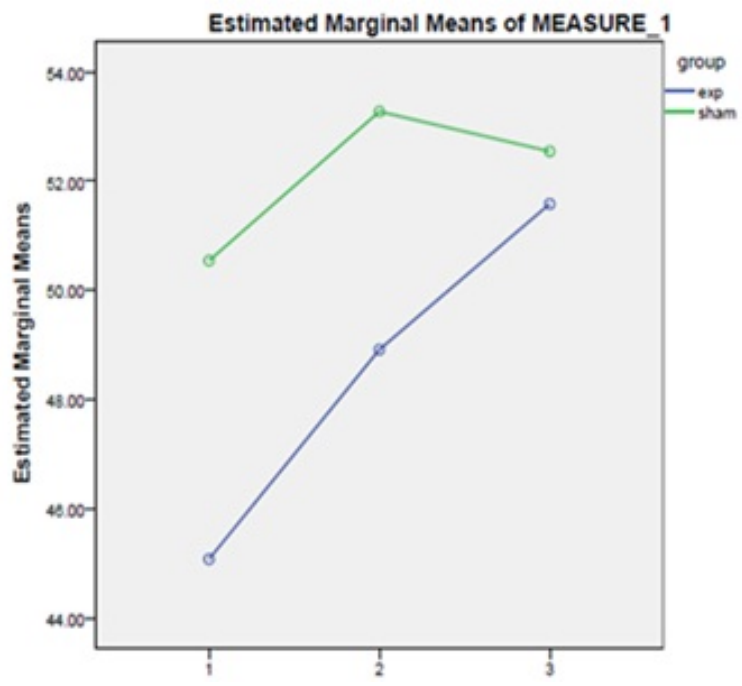

Figure 1 Groups interaction and behavior differences between 2 groups in Berg Balance Scale.

\section{Discussion}

Parkinson's disease is a progressive neurodegenerative disorder characterized by motor and non-motor symptoms [1]. Postural instability is one of the four cardinal symptoms of PD [3] which may be present sub clinically in the early stage of the disease [5]. It is known that the executive functions and attention supported by the DLPFC play a key role in walking speed, especially in the elderly and frontal areas are functional during locomotion [20]. TDCS may modulate prefrontal dopamine release, thus affecting motor performance [11].

Preliminary findings showed benefits of TDCS in multiple aspects of PD such as working memory, cognitive ability, motor function, walking ability and freezing of gait and fatigue [9-14]. Motor rehabilitation may be regarded as a process of relearning how to move to satisfy personal needs; practice and training lead to effective motor learning thus improving skills and motor performance. PD subjects demonstrate a relatively preserved ability in motor learning, but consolidation of learned material is defective and translation to the clinical setting may be critical [21].

General physiotherapy (stretching, muscle strengthening, balance and postural exercises), occupational therapy, and treadmill training, are frequently adopted to improve specific aspects of mobility [20]. Occupational therapy is a conventional effective treatment in motor function improvement and fall prevention in patients with $P D$ performed from the past till to date $[22,23]$.

Results of this study showed, the main effect of time was significant in both groups and there was a significant difference between before and just after therapeutic course termination, but behavior of groups didn't differ regarding changes in BBS at any time point. Therefore, TDCS cannot have any additive effect than which has occupational therapy as the common effective intervention. In line with this study, CostaRibeiro et al. [17] compared the effects of a-TDCS (anode placed in $\mathrm{Cz}$ ) combined with gait training versus sham-TDCS combined with training on functional mobility of individuals with PD. Both groups displayed similar improvements on gaitrelated outcome measures. However, in another study, combining TDCS on primary motor and premotor cortex with physical training showed positive effects on gait and balance in patients with PD but TDCS alone did not [18].

In contrast, a recent study showed the acute positive effect of left dorsolateral prefrontal cortex (DLPFC) TDCS on balance and functional mobility over to sham- TDCS measured by Berg Balance Scale, Dynamic Gait Index and Timed Up and Go. Participants in this study were Seventeen patients with PD who all received one session of two conditions (TDCS and sham- TDCS) at least $48 \mathrm{~h}$ apart [19].

\section{Conclusion}

Although the findings of this pilot study couldn't show positive effects of TDCS on function balance in patients with $\mathrm{PD}$, but one explanation may be due to high score of baseline BBS in our patients. Future studies with more sample size and severe balance dysfunction and longer follow up duration should be conducted. Furthermore, using objective measures of balance by force plate may be useful in detection of minimal changes especially in patients with subclinical balance dysfunction. 
Based on the results of the present study there is no significant effect of TDCS on functional balance in patients with PD at any time point.

\section{Conflict of Interest}

The authors declare that they have no conflict of interest.

\section{Funding}

Authors have no funding source.

\section{References}

1. Leite J, Gonçalves OF, Carvalho S (2014) Facilitative effects of bihemispheric TDCS in cognitive deficits of Parkinson disease patients. Medical hypotheses 82: 138-140.

2. Jankovic J (2008) Parkinson's disease: Clinical features and diagnosis. Journal of Neurology, Neurosurgery \& Psychiatry 79: 368-376.

3. Chastan N, Debono B, Maltête D, Weber J (2008) Discordance between measured postural instability and absence of clinical symptoms in Parkinson's disease patients in the early stages of the disease. Movement Disorders 23: 366-372.

4. Kerr GK, Worringham CJ, Cole MH, Lacherez PF, Wood JM, et al. (2010) Predictors of future falls in Parkinson disease. Neurology 75: $116-124$

5. Park JH, Kang YJ, Horak FB (2015) What is wrong with balance in Parkinson's disease? Journal of Movement Disorders 8: 109.

6. Adkin A L, Frank JS, Jog MS (2003) Fear of falling and postural control in Parkinson's disease. Journal of Movement Disorders 18: $496-502$

7. Varanese S, Birnbaum Z, Rossi R, Di-Rocco A (2010) Treatment of advanced Parkinson's disease. Parkinson's Disease.

8. Ferrucci $\mathrm{R}$, Cortese $\mathrm{F}$, Bianchi $\mathrm{M}$, Pittera $\mathrm{D}$, Turrone $\mathrm{R}$, et al. (2016) Cerebellar and motor cortical transcranial stimulation decrease levodopa-induced dyskinesias in Parkinson's disease. The Cerebellum 15: 43-47.

9. Benninger DH, Mikhail L, Grisel L, Natassja P, David AL, et al. (2011) Transcranial direct current stimulation for the treatment of focal hand dystonia. Movement Disorders 26: 1698-1702.

10. Rosa M, Michela B, Sandra R, Italo O, Clarissa F, et al. (2014) Time up and go task performance improves after transcranial direct current stimulation in patient affected by Parkinson's disease. Neuroscience letters 580: 74-77.

11. Paulo SB, Roberta F, Sergio PR, Priscila C, Michael N, et al. (2006) Effects of transcranial direct current stimulation on working memory in patients with Parkinson's disease. Journal of the neurological sciences 249: 31-38.

12. Francesca V, Giuseppe C, Filippo B, Nicolò GP, Giorgio S, et al. (2014) Transcranial direct current stimulation for treatment of freezing of gait: A cross-over study. Movement Disorders 29: 1064-1069.

13. Deniz D, Zachary G, Gabriela LB, Alvaro PL, Felipe F (2014) Effects of TDCS on executive function in Parkinson's disease. Neuroscience letters 582: 27-31.

14. Bijan F, Maryam R, Amin A, Mohammad RM, Seyed PM, et al. (2017) Repeated sessions of transcranial direct current stimulation evaluation on fatigue and daytime sleepiness in Parkinson's disease. Neurological Sciences 38: 249-254.

15. Sanne B, Evelien N, Elke H, Griet V, Raf M, et al. (2015) Transcranial direct current stimulation in Parkinson's disease: Neurophysiological mechanisms and behavioral effects. Neuroscience \& Biobehavioral Reviews 57: 105-117.

16. Jean-Jacques OX, Reza S (2014) Electrifying the motor engram: Effects of tDCS on motor learning and control. Experimental Brain Research 232: 3379-3395.

17. Adriana C, Ariadne M, Thamyris B, Yumi A, Rebeca $C$, et al. (2017) Transcranial direct current stimulation associated with gait training in Parkinson's disease: a pilot randomized clinical trial. Developmental Neurorehabilitation 20: 121-128.

18. Kaski D, Dominguez RO, Allum JH, Islam AF, Bronstein AM (2014) Combining physical training with transcranial direct current stimulation to improve gait in Parkinson's disease: a pilot randomized controlled study. Clinical Rehabilitation 28: $1115-1124$.

19. Eduardo L, Samara SC, Carlos C, Aldair JO, Sérgio M, et al. (2017) Can transcranial direct current stimulation on the dorsolateral prefrontal cortex improves balance and functional mobility in Parkinson's disease? Neuroscience Letters 636: 165-169.

20. Alesandro B, Stefano V, Giovanni Z, Jack MG, Stefania B, et al. (2005) Executive function correlates with walking speed in older persons: the InCHIANTI study. Journal of the American Geriatrics Society 53: 410-415.

21. Giovanni A, Roberta M, Laura A, Elisa P (2016) Rehabilitation for Parkinson's disease: Current outlook and future challenges. Parkinsonism \& Related Disorders 22: 560-564.

22. Bijan F, Maryam R, Amin A, Mohammad RM, Seyed PM, et al. (2005) Gait and step training to reduce falls in Parkinson's disease. Neuro Rehab 20: 183-190.

23. Murphy S, Tickle-Degnen L (2001) The effectiveness of occupational therapy-related treatments for persons with Parkinson's disease: A meta-analytic review. American Journal of Occupational Therapy 55: 385-392. 